\title{
PROPIEDADES FÍSICAS Y MECÁNICAS DE LADRILLOS MACIZOS CERÁMICOS PARA MAMPOSTERÍA
}

\author{
MECHANICAL \& PHYSICAL PROPERTIES OF SOLID, MASONRY CERAMIC BRICKS
}

Nelson Afanador García

Ing. Civil, Ms.C., Docente Asistente, Facultad de Ingeniería, Investigador

Línea Materiales y Sísmica

Universidad Francisco de Paula Santander, Ocaña - Colombia.

nafanadorg@ufpso.edu.co

Gustavo Guerrero Gómez

Ing. Mecánico, Docente Auxiliar, Facultad de Ingeniería, Investigador Grupo GITYD

Universidad Francisco de Paula Santander, Ocaña - Colombia.

gguerrerog@ufpso.edu.co

Richard Monroy Sepúlveda

Ing. de Producción Industrial, Docente catedrático, Facultad de Ingeniería, Investigador Grupo

GITEC Universidad Francisco de Paula Santander, Cúcuta - Colombia.

cimac@motilon.ufps.edu.co

Fecha de recepción: 6 de diciembre de 2011

Fecha de aprobación: 15 de mayo de 2012

\section{RESUMEN}

El presente artículo es el resultado de evaluar las propiedades mecánicas de los ladrillos macizos cerámicos fabricados a mano en el municipio de Ocaña, aplicando en primera instancia ensayos de caracterización física de la arcilla empleada como materia prima, para luego realizar pruebas de control de calidad no destructivas y destructivas de los ladrillos de mampostería que se seleccionaron entre los diferentes chircales o unidades productivas del sector, utilizando para tal fin, la norma técnica Colombiana NTC-4017 [16], “Métodos para muestreo y ensayos de unidades de mampostería y otros productos de arcilla", que permitieron conocer las características y propiedades de los materiales que integran los elementos estructurales, analizando principalmente el módulo de elasticidad y la resistencia a la compresión, como parámetros que influyen en forma directa en la rigidez de la edificación y que concuerdan con las investigaciones de Hemant B. et al[11] y Takeuchi C. [20], bajo el Código Colombiano de Construcción Sismo Resistente de 2010 (NSR-10) [4].

Palabras clave: mampostería, ladrillos, propiedades de los ladrillos, resistencia en ladrillos. 


\begin{abstract}
This paper results of evaluating mechanical properties of hand-made, ceramic solid bricks in Ocaña town, after applying physical characterization tests of clay used as a raw material for subsequent non-destructive \& destructive quality control testing selected from different brickworks or production units. That test was based on "Methods for masonry sampling and testing units and other products clay", a Colombian technical standard NTC-4017 [16], which let to know material characteristics and properties of structural elements by analyzing mainly elastic and compressive strength module as parameters with direct influence upon building stiffness (see Hemant B. et al [1 1] y Takeuchi C. [20]) covered by Colombian Earthquake Resistant Construction Code, 2010 (NSR-10) [4].
\end{abstract}

Keywords: bricks, brick properties, brick toughness, masonry.

\title{
INTRODUCCIÓN
}

Los ladrillos toletes macizos de arcilla cocidos se utilizan en la construcción de obras civiles principalmente en edificios y viviendas, por los bajos costos del material, su propiedad de aislamiento térmico y su disponibilidad geográfica. Estas edificaciones están diseñadas en su mayoría, con un sistema de pórticos resistentes a momentos, mampostería reforzada o mampostería confinada, según el reglamento colombiano de construcción sismo resistente (NSR-10) [4].

Durante su vida útil, estas construcciones están sujetas a la acción de cargas verticales y horizontales, y pueden generar fracturas en piezas de mampostería y en muros. Si su calidad no es adecuada, pone en peligro a sus ocupantes, y este fracturamiento influye en un aumento de su vulnerabilidad, en especial por la acción sísmica [6, 3].

Esta investigación realizada por Afanador G. N. [1], tuvo como objetivo determinar la resistencia a la fractura en ladrillos cerámicos macizos fabricados manualmente, aplicando los ensayos de resistencia mecánica a la compresión y a la flexión, bajo la norma técnica colombiana NTC-4017 determinando sus valores máximos y mínimos, y la probabilidad de falla del ladrillo cerámico sujeto a la resistencia mecánica $[8,15]$. Adicionalmente, se calculó la tasa inicial de absorción y la absorción final con pruebas estandarizadas para ladrillos producidos en Colombia [16], junto con la caracterización de la arcilla del municipio de Ocaña utilizada en la elaboración de los ladrillos. Estos resultados permitieron proponer un valor a la resistencia a la compresión de muros f' ${ }_{m}$ (con fines de diseño), usando la resistencia a la compresión del ladrillo macizo de arcilla cocida y la resistencia a la compresión del mortero de pega. 


\section{MATERIALES Y MÉTODOS}

El área de estudio fue el municipio de Ocaña bajo las coordenadas geográficas $X=1.077 .500$ a 1.082.000 y $Y=1.401 .000$ a 1.407.000. La extensión aproximada del área urbana es de 7.74 $\mathrm{Km}^{2}$. En el Municipio, se encontraron 16 chircales activos ubicados en diferentes zonas del casco urbano de la ciudad, que producen cerca de 352.000 ladrillos/mes (según encuesta realizada), Figura 1 [2], los cuales emplean procesos manuales y rudimentarios en las diferentes etapas para la fabricación de estas piezas de mampostería. El estudio abarco los 16 chircales que rodean la ciudad, en los cuales se tomaron 165 ladrillos por chircal, para un total de 2.640 ladrillos. Se usaron 33 ladrillos para realizarle cuatro ensayos que son: resistencia a la compresión, resistencia a la flexión, tasa inicial de absorción, y absorción de agua.

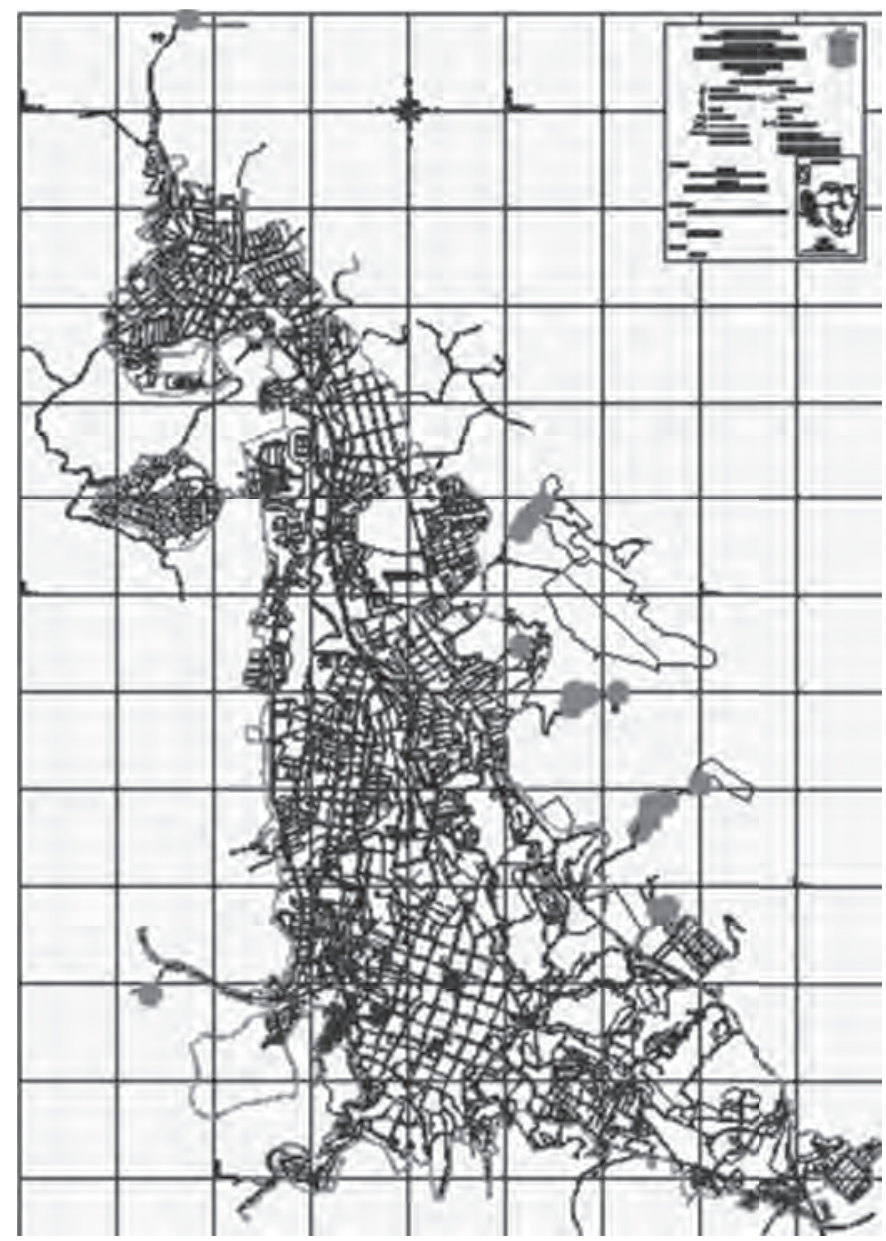

Figura 1. Ubicación de chircales.

Fuente: Alcaldía municipal de Ocaña [2] 
Se hizo una clasificación del suelo para establecer los porcentajes de arenas, limos y arcillas utilizado en la materia prima para elaborar los ladrillos cerámicos. Como caso de estudio, se tomó el chircal 1. La toma de la muestra se hizo mediante un muestreo sistemático por el método de cuarteos sucesivos [17] hasta obtener 4 kg, para realizar los ensayos de caracterización física de la muestra desde el punto de vista de: clasificación granulométrica [13], medidas de plasticidad por el método de los límites de Atterberg [14], y clasificación del porcentaje de arcillas y limos por el método de análisis granulométrico por medio de hidrómetro [18]. Para el manejo de los datos, se utilizó una hoja de cálculo en el programa Microsoft Excel, con el reporte dado por la máquina Universal de ensayos tipo Pinzuar PU-100 y el software estadístico Statgraphics.

La metodología utilizada para determinar las propiedades físicas y mecánicas de los ladrillos fue tomada de la NTC 4017, Métodos para muestreo y ensayos de unidades de mampostería y otros productos de arcilla [16], en donde se expone, los procedimientos de selección y preparación de la muestra.

La resistencia a la compresión de ladrillo de arcilla, $f^{\prime}{ }_{\text {cu, }}$ se usa como control de calidad en la elaboración (dosificación de los materiales, temperatura y tiempo de horneado), para conocer la calidad de los materiales y su materia prima utilizados en la fabricación de ladrillo y para encontrar la resistencia a la compresión de la mampostería $f^{\prime}{ }_{m}$ a partir de fórmulas que relacionan las propiedades de la unidad y los morteros [20].

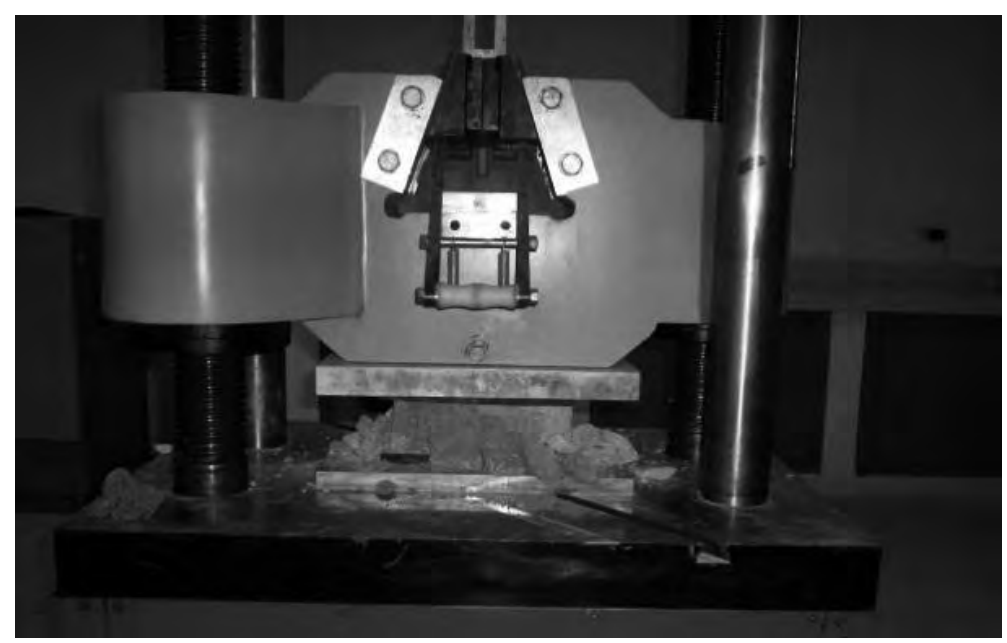

Figura 2. Ensayo de resistencia a la compresión en ladrillos (Laboratorio de resistencia de materiales y sísmica Universidad Francisco de Paula Santander, Ocaña, UFPSO)

El ensayo de resistencia a la compresión (Figura 2), consistió en llevar la pieza de ladrillo a la falla y registrar la carga de rotura en el área de contacto, para determinar el esfuerzo de compresión máximo [9] mediante la ecuación 1. 


$$
\mathrm{f}^{\prime}{ }^{\prime}=\frac{\mathrm{W}}{\mathrm{A}}
$$

Dónde:

$\mathrm{f}^{\prime}{ }_{\mathrm{cu}}=$ resistencia a la compresión de ladrillo Pa $\times 10^{4}\left(\mathrm{o} \mathrm{Kgf/ \textrm {cm } ^ { 2 } )}\right.$

$W=$ carga máxima (de rotura) en $\mathrm{N}$ (o Kgf)

$A=$ promedio área brutas de las superficies superior e inferior del espécimen en $\mathrm{cm}^{2}$.

El módulo de rotura (ensayo de flexión) es una propiedad importante como criterio de durabilidad y para entender el mecanismo de falla de la mampostería cuando se solicitan esfuerzos de compresión y flexión, casos muy comunes en la mampostería. El ladrillo fue sometido a una carga puntual en el centro de la pieza con una velocidad de carga inferior a 1.3 mm/mín (Figura 3). Registrando la carga de falla y reemplazando en la expresión (2), se obtiene el módulo de rotura (MR).

$$
\mathrm{MR}=3 \mathrm{~W}\left(\frac{\mathrm{L}}{2}-\mathrm{X}\right) / \mathrm{bd}^{2}
$$

Donde:

$\mathrm{W}=$ carga máxima de rotura en $\mathrm{N}$ (o Kgf)

$\mathrm{L}=$ distancia entre los soportes en $\mathrm{mm}$

$\mathrm{b}=$ ancho neto de la muestra en el plano de falla en $\mathrm{mm}$

$\mathrm{d}=$ profundidad de la muestra en el plano de falla en $\mathrm{mm}$

$X=$ distancia promedio del plano de falla al centro de la pieza, medida en la dirección de la línea central de la superficie sometida a tensión en mm

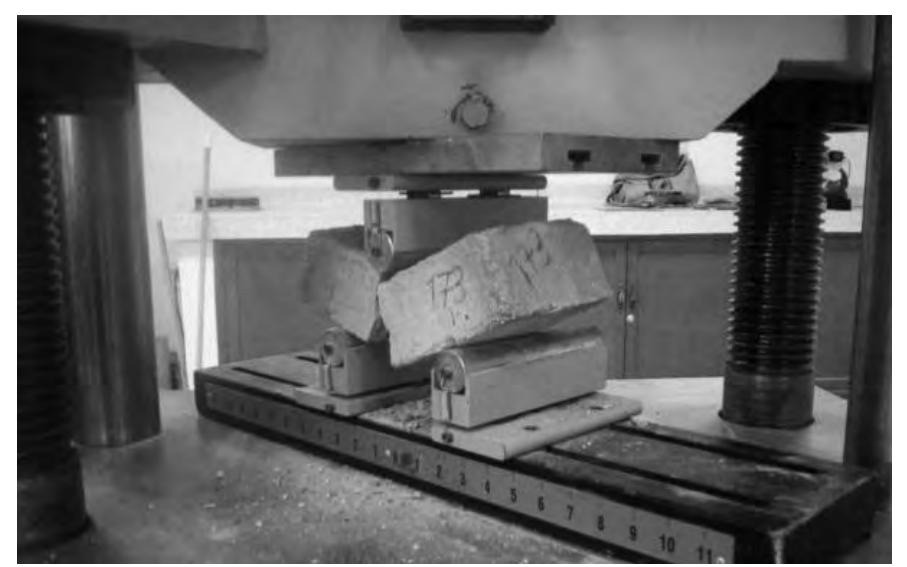

Figura 3. Ensayo de flexión en ladrillos

(Laboratorio de resistencia de materiales y sísmica UFPSO)

Entre las propiedades físicas que se estudiaron en los ladrillos se encuentran, la tasa inicial de absorción y la absorción de agua. La tasa inicial de absorción (TIA), dada en g/ $\mathrm{cm}^{2} / \mathrm{min}$, mide la cantidad de agua que absorbe el ladrillo en un minuto, pues los poros de los ladrillos funcionan 
como capilares en presencia de agua. Cuando se coloca el mortero de pega o de relleno de las unidades, succiona parte del agua del mortero, que afecta su adherencia y la consistencia del mortero. Una adherencia deficiente afecta la resistencia de la mampostería, la durabilidad y penetración de agua [20]. El ensayo consistió en determinar la masa seca y la masa final del ensayo (Figura 4), registrando la TIA que se calculó con la ecuación (3).

$$
\text { T.I.A. }=\frac{\text { Masa }_{\text {final }}-\text { Masa }_{\text {final }}}{\text { Área }_{\text {contacto con el agua }}}
$$

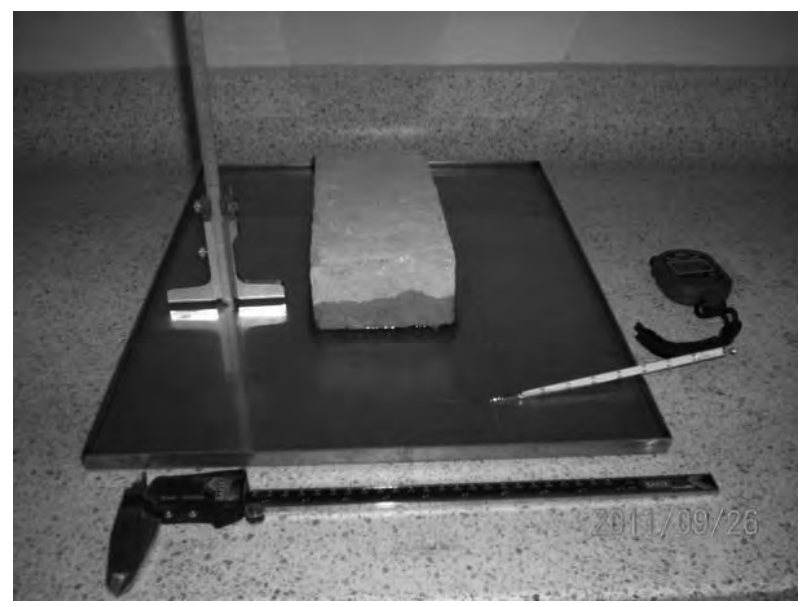

Figura 4. Ensayo de Tasa inicial de absorción en ladrillos. (Laboratorio Resistencia de materiales y sísmica UFPSO)

La absorción de agua afecta la durabilidad de la unidad y la mampostería. Si la unidad tiene absorción alta, puede presentar cambios volumétricos significativos o permeabilidad alta a la penetración de agua, y puede causar decoloraciones. Este ensayo consiste en obtener la masa sumergida en agua durante 24 horas de inmersión y restarle la masa seca; esta diferencia se expresa en porcentaje de masa seca (4).

$$
\% \text { absorción }=\frac{\left(\mathrm{W}_{\mathrm{ss}}-\mathrm{W}_{\mathrm{s}}\right)}{\mathrm{W}_{\mathrm{s}}} * 100
$$

Donde:

$W_{s s}=$ masa sumergida en agua de ladrillo saturado luego de la inmersión en agua fría, en $g$. $\mathrm{W}_{\mathrm{s}}=$ masa seca del espécimen antes de inmersión, en $\mathrm{g}$.

\section{RESULTADOS Y ANÁLISIS}

A la muestra tomada en el chircal 1 se le aplico el ensayo granulométrico y de acuerdo con la USCS se clasifica como CL, es decir arcillas inorgánicas de plasticidad baja a media, arcillas 
arenosas o arcillas pobres, mientras que con la clasificación de la AASHTON se clasifica como A-6 (13), es decir, suelo fino donde más del 35\% de la muestra total pasa por la malla 200, con un 3.28\% de grava, $22.85 \%$ de arenas y un $73.87 \%$ de finos (Figura 5), y las medidas de plasticidad por el método de los límites de Atterberg indicaron un límite liquido de 36.01\%, un límite de plasticidad de 16.6\%, un índice de plasticidad de 19\% y humedad natural de un 15.09\%; y la clasificación de arcillas y limos por el método de análisis granulométrico por medio del hidrómetro, utilizando como agente dispersante una solución de sodio esametafosfato al 4\% en $125 \mathrm{ml}$ de agua, clasificó el 43\% como limos, el 38\% como arena y el 19\% restante como arcillas.

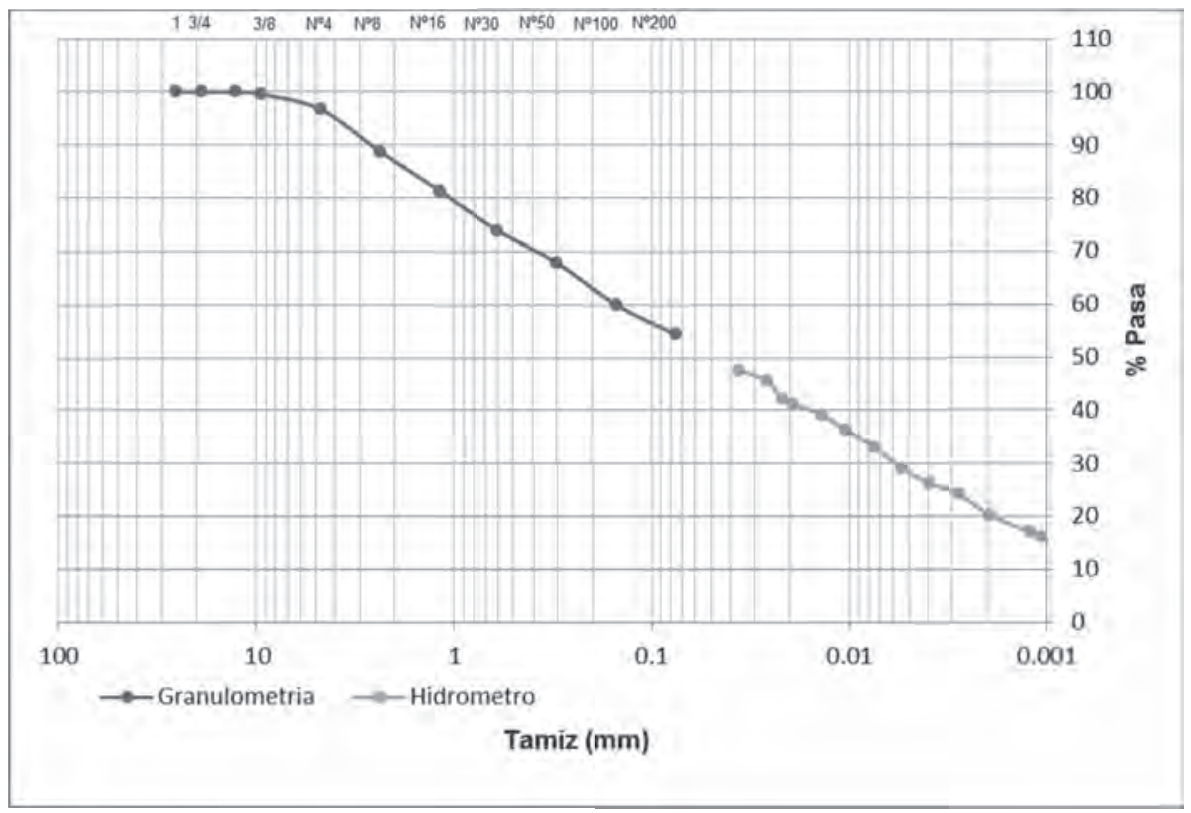

Figura 5. Ensayo de granulometría por tamizado y por hidrómetro [1]

En el ensayo de resistencia a la flexión, se presentan los resultados de 16 chircales que corresponden a valores promedio de una muestra de 32 ladrillos ensayados para un total de 512 ladrillos de arcilla cocida, usando la metodología NTC 4017, donde el chircal 15 tiene el valor máximo observado de $1.63 \mathrm{MPa}$, como se ve en la Figura 6.

Si se tiene en cuenta que la resistencia a la flexión varía entre el 10 al 30\% de la resistencia a la compresión según $[20,15]$, y que la resistencia a la compresión mínima es de 10MPa para una unidad y de 14MPa para cinco unidades de ladrillo, se puede establecer un intervalo de resistencia mínimo a la flexión de 1.0 a 1.4MPa. Por lo tanto, los chircales 1, 7, 8, 9, 10, 14 y 15 cumplen con el objetivo de resistencia mínima a la flexión y estos chircales representan tan sólo el 44\% de los chircales que rodean la ciudad. 


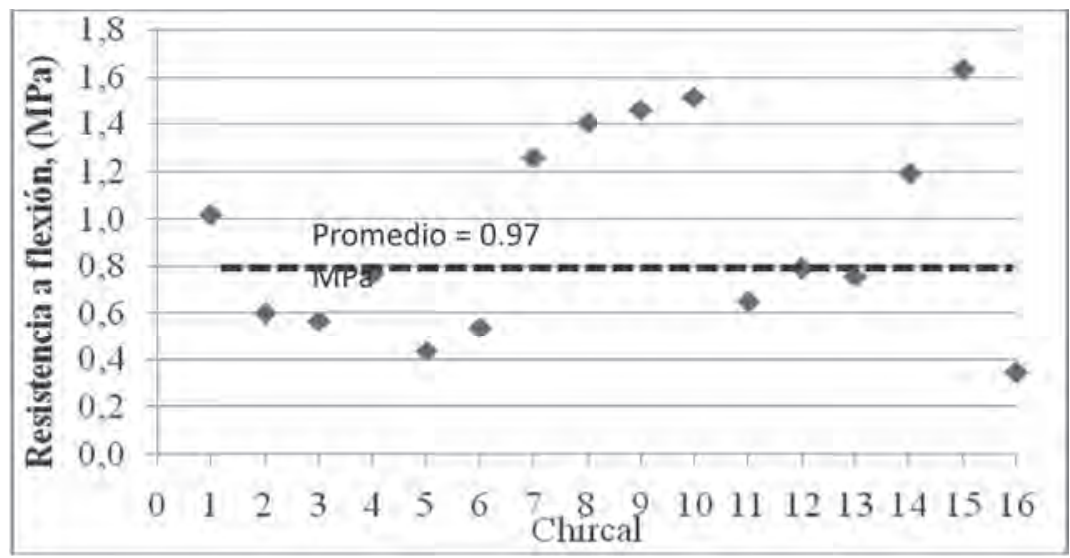

Figura 6. Resistencia a la flexión en ladrillos.

Fuente: Afanador G.N. [1]

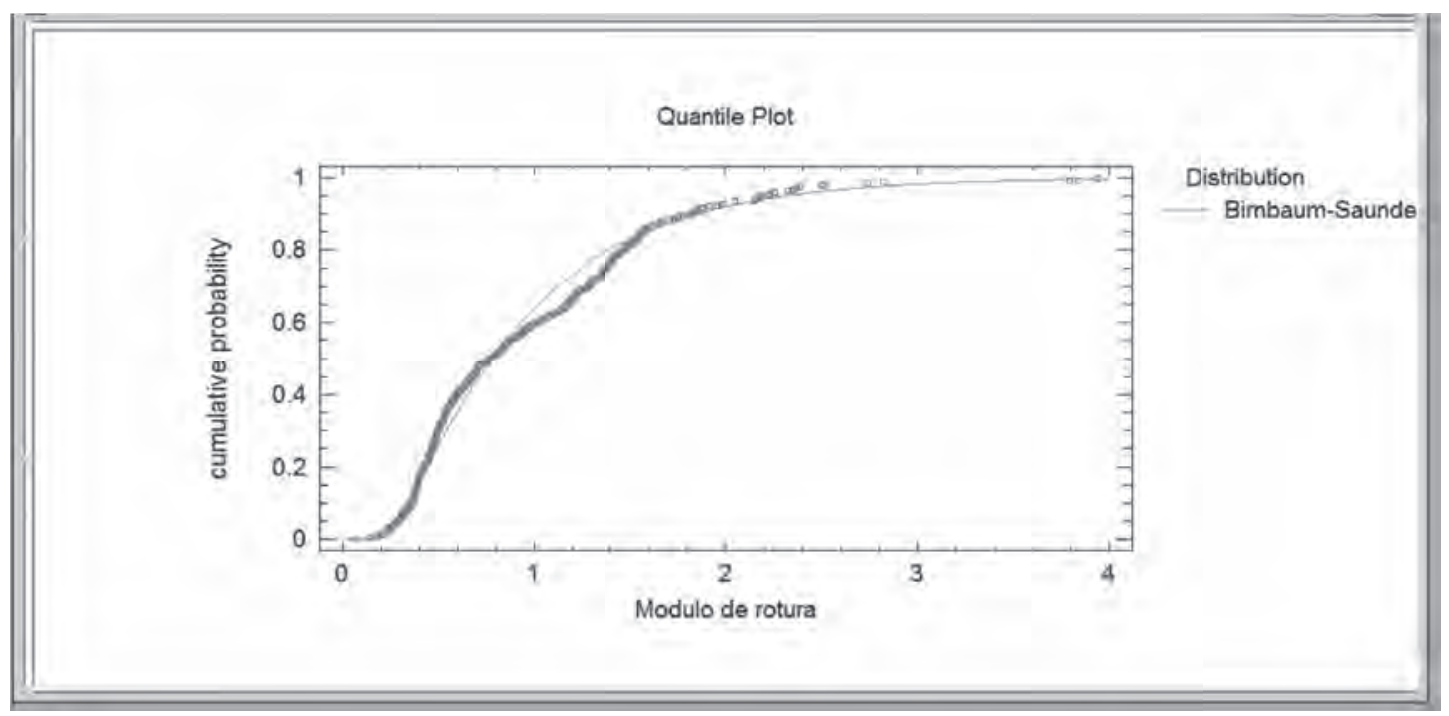

Figura 7. Distribución de probabilidad de Birnbaum-Saunders flexión en ladrillos [1]

Al realizar un análisis estadístico a los 512 datos obtenidos experimentalmente del ensayo a flexión, se encontró que la distribución de probabilidad que mejor se ajustó fue la distribución de Birnbaum - Saunders [5], con un factor de forma $=0.6973$ y un factor de escala $=0.7821$ (Figura 7), donde la ordena es el esfuerzo a la compresión y la abscisa es la probabilidad de falla. La distribución de Birnbaum - Saunders es una distribución utilizada en el estudio de vibraciones en aviones comerciales y en los problemas de fatiga de materiales [7] y fue la que mejor se adaptó a los datos obtenidos. 


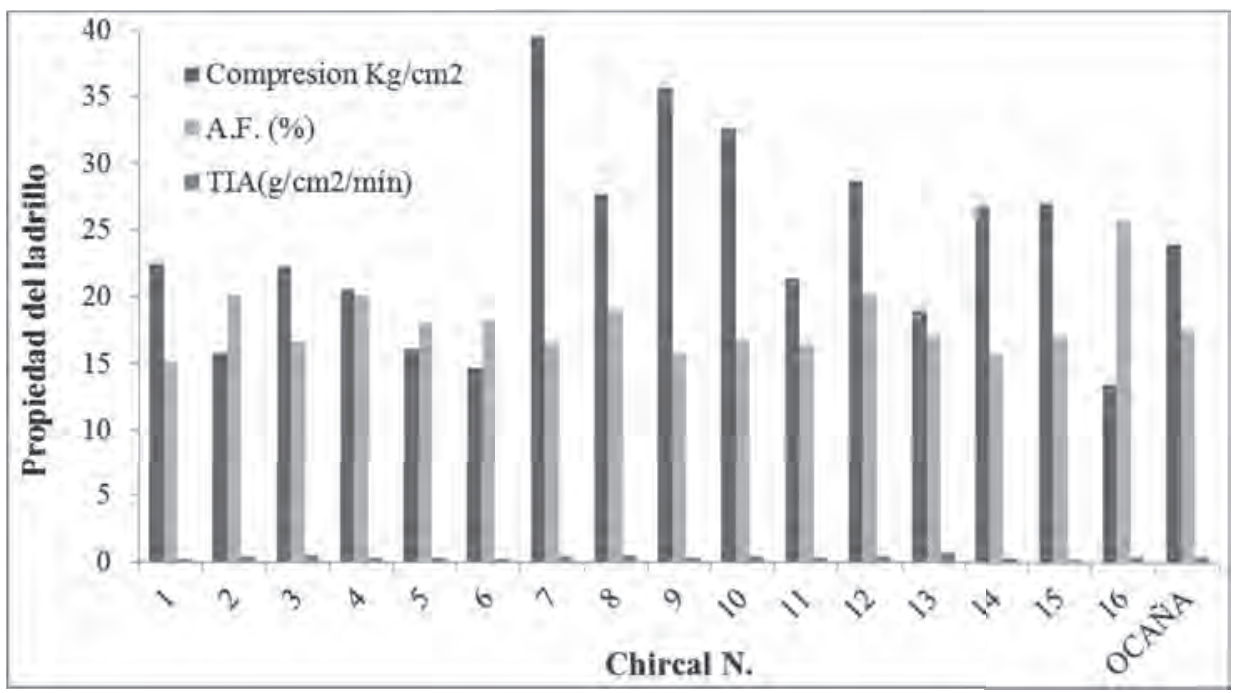

Figura 8. Resistencia a la compresión, absorción de agua y tasa inicial de absorción [1].

La Figura 8 muestra la variación de la resistencia a la compresión ( $f^{\prime}{ }_{c u}$ ), absorción final (AF) y tasa inicial de absorción (TIA), y en la Tabla 1, se dan los datos con el coeficiente de variación y el promedio. La resistencia a la compresión en piezas de ladrillo tolete macizo de arcilla cocida ( $f^{\prime}{ }^{\prime}$ ) tiene una variación en la resistencia que oscila entre 13.36 a $39.50 \mathrm{Kg} / \mathrm{cm}^{2}$ (promedio 23.93 MPa, coeficiente de variación (COV, 0.56). Se encontró que la AF varía entre 15.20 a 25.60\% (promedio de $17.41 \%$, COV 0.16), que indica una alta absorción de agua de la pieza sumergida por 24 horas. La NTC 4205 establece para paredes interiores, un máximo de 17\% y para paredes exteriores un máximo de 13.5\%, lo cual indica una falta de cocción. La TIA fluctúa entre 0.19 a $0.74 \mathrm{~g} / \mathrm{cm}^{2} / \mathrm{mín}^{2}$ (promedio de $0.39 \mathrm{~g} / \mathrm{cm}^{2} / \mathrm{mín}$ COV 0.45). Estos valores exceden los permitidos por [15] ya que la máxima TIA es de $0.25 \mathrm{~g} / \mathrm{cm}^{2} / \mathrm{mín}_{\text {. }}$

El fabricante 7 ofrece los mejores resultados en la resistencia a la compresión (Tabla 1), de 39.50 MPa que es superior al promedio de $23.93 \mathrm{MPa}$, y representa el $28.21 \%$ del valor esperado para el promedio de cinco unidades (140MPa); según [15], una absorción final promedio (AF) a las 24 horas de inmersión en agua de 16.56\% (ligeramente inferior al promedio observado de 17.41\%), que es un valor aceptable [15]. Además, cuenta con una tasa inicial de absorción de $0.399 \mathrm{~g} /$ $\mathrm{cm}^{2} / \mathrm{mín}$ (promedio de $0.387 \mathrm{~g} / \mathrm{cm}^{2} / \mathrm{mín}$ ), muy superior al esperado [15], y una variación en la TIA

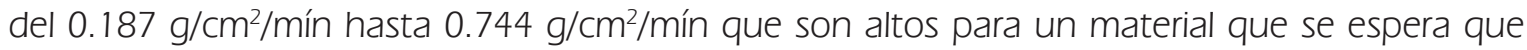
no absorba el agua del mortero y permita el fraguado del mortero en forma normal. Los demás fabricantes tienen valores inferiores de la resistencia a la compresión, según los estándares de calidad establecidos para este material, junto con la TIA y AF. 
Tabla 1. Resumen de pruebas sobre ladrillos. [1]

\begin{tabular}{|c|c|c|c|c|c|c|}
\hline Chircal N. & \multicolumn{2}{|c|}{$f_{c u}^{\prime}\left(\mathrm{Kg} / \mathrm{cm}^{2}\right)$} & \multicolumn{2}{|c|}{ A.F. (\%) } & \multicolumn{2}{|c|}{ T.I.A.(g/ $\left./ \mathrm{cm}^{2} / \mathrm{min}\right)$} \\
\hline 1 (32 Lad.) & 22.46 & {$\left[0.34^{\mathrm{q}}\right]$} & 15.20 & {$[0.22]$} & 0.2604 & {$[0.2$} \\
\hline 2132 & $\angle$ & 3] & 20.21 & {$[0.06]$} & 0.4101 & {$[0.2$} \\
\hline 31 & 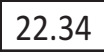 & $2]$ & 16.57 & 71 & 93 & {$[0$.} \\
\hline 41 & 20.56 & 0.53 & 20.09 & 2 & 66 & [0.26] \\
\hline 51 & $y$ & {$[0.20]$} & 18.16 & $12]$ & 306 & {$[0.39]$} \\
\hline 613 & 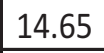 & [0.28] & 18.20 & {$[0.06]$} & 96 & {$[0.21]$} \\
\hline 71 & 35 & {$[0.88]$} & 16.56 & [0.11] & 33 & U. \\
\hline 81 & 21 & {$[0.14]$} & 19.02 & {$[0.06]$} & 50 & {$[0.18$} \\
\hline 91 & 35. & [0.24] & 15.80 & [0.07] & 93 & {$[0.58]$} \\
\hline $10(3$ & 32 & {$[0.36]$} & 16.79 & [0.07] & 07 & {$[0.24]$} \\
\hline $11(3$ & 21.37 & {$[0.30]$} & 16.42 & [0.10] & 0.3211 & [0.35] \\
\hline 123 & 28.66 & {$[0.30]$} & 20.21 & [0.06] & 0.3932 & {$[0.29]$} \\
\hline $13(32$ & 18.93 & {$[0.32]$} & 17.16 & {$[0.12]$} & 0.7438 & {$[0.16]$} \\
\hline $14(3)$ & 26.74 & [0.49] & 15.62 & [0.12] & 306 & {$[0.39]$} \\
\hline $15(3)$ & 26.96 & {$[0.56]$} & 17.00 & {$[0.16]$} & 72 & $0.26]$ \\
\hline $16(32 \mathrm{~L}$ & 13.34 & [0.13] & 25.60 & {$[0.03]$} & 0.3862 & {$[0.15]$} \\
\hline CAÑA (512 L) & 23.93 & {$[0.56]$} & 17.41 & {$[0.16]$} & 0.3866 & {$[0.45]$} \\
\hline
\end{tabular}

${ }^{a}$ En la Tabla 1. [] Indica coeficiente de variación
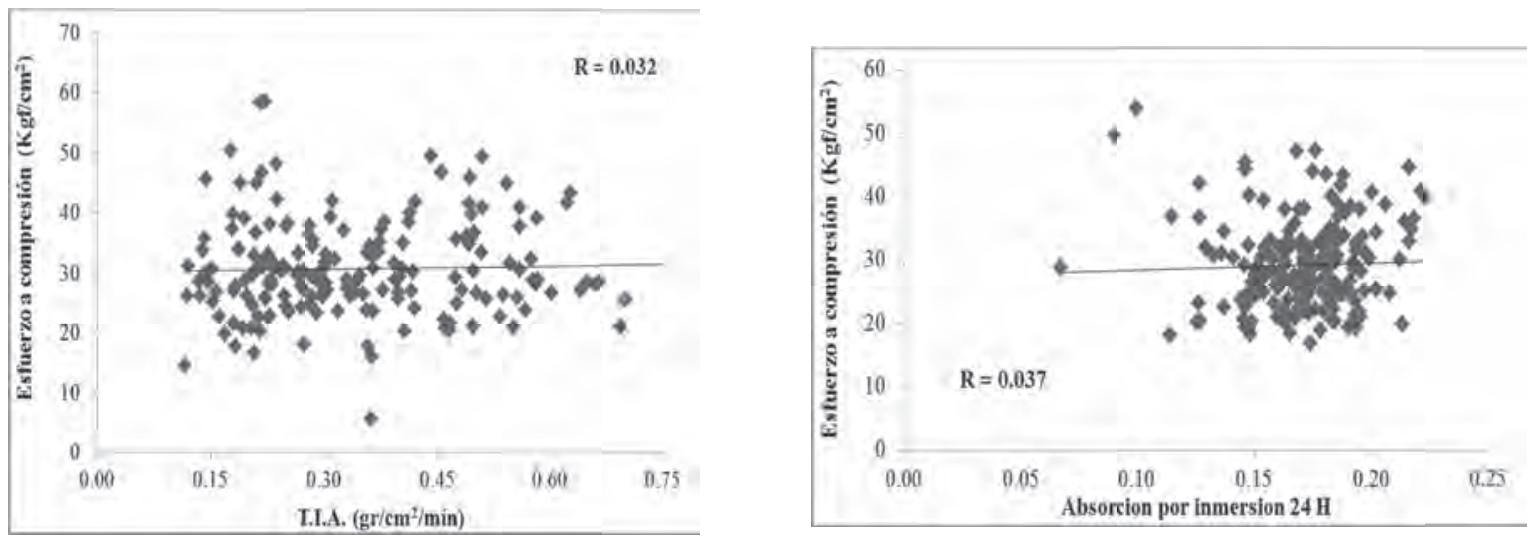

Figura 9. Efecto de absorción por inmersión $24 \mathrm{H}$ y la tasa inicial de absorción sobre la resistencia a la compresión ( $\mathrm{R}=$ coeficiente de correlación) [1]

Se estableció una correlación de los resultados de los ensayos de tasa inicial de absorción (TIA), y absorción por inmersión 24 horas (AF), con la resistencia a la compresión bajo fuerzas 
compresivas concéntricas uniaxiales, Figura 9 [1]. Tanto para valores bajos como altos de TIA, se encontraron valores bajos y altos de la resistencia a la compresión $f^{\prime}{ }_{c u}$. Para el presente trabajo, no se observó alguna correlación entre la resistencia a la compresión, la TIA y la absorción por inmersión en agua (24H), muy diferente a las relaciones encontradas entre $f^{\prime}{ }_{c u}$ Vs. TIA y $f^{\prime}{ }_{c u}$ Vs. AF con coeficientes de correlación de -0.77 y -0.24 respectivamente, muy similar a la encontrada en otras investigaciones como en Kaushik Hemant B., et al [11].

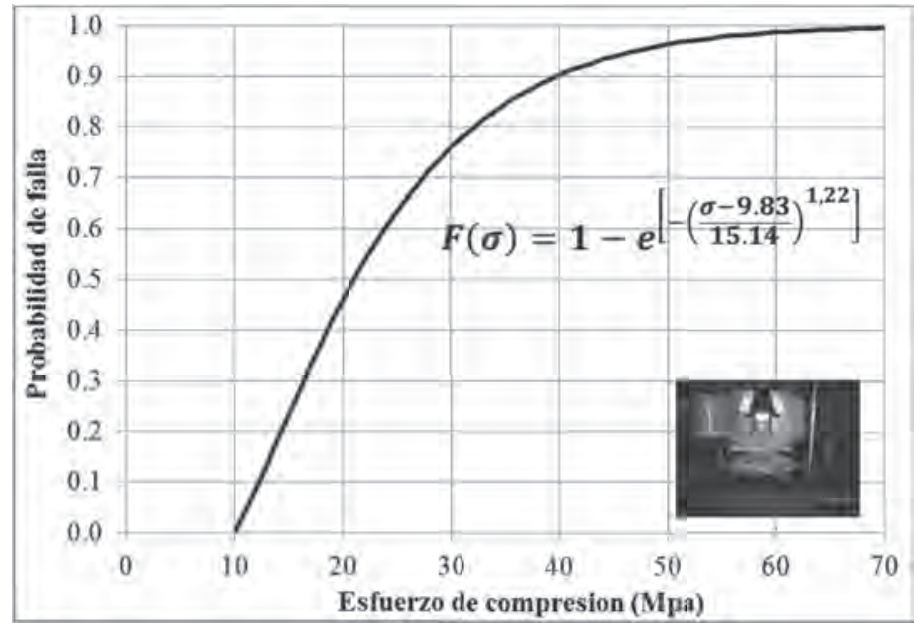

Figura 10. Función distribución acumulativa F(o) Weibull de tres parámetros, Ocaña [1]

Los resultados obtenidos para el ensayo de compresión en ladrillos macizos de arcilla cocida obedecen a una distribución de probabilidad de Weibull de tres parámetros [19], Figura 10, donde el parámetro de forma (b) es 1.224, el parámetro de escala (q) es 15.1427, el parámetro de localización (d) es 9.8273 y donde la ecuación de la distribución de Weibull de tres parámetros para todos los ensayos de resistencia a la compresión, es la que se encuentra en la Figura 9, lo cual permite estimar para un esfuerzo de trabajo de 40 MPa una probabilidad de falla del 90\% aproximadamente, alta probabilidad para un esfuerzo tan bajo.

Es posible establecer una correlación entre la resistencia especificada a la compresión de la mampostería $f^{\prime}{ }_{m}$ y la resistencia a la compresión uniaxial a compresión del ladrillo de arcilla cocido

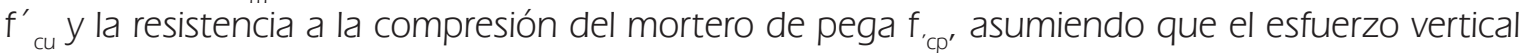
del mortero de pega es igual a la resistencia máxima del mortero bajo un estado de compresión triaxial y que para las unidades de ladrillo, es válido el criterio de falla lineal de Mohr Coulomb, cuando están solicitadas a esfuerzos verticales de compresión y biaxiales de tensión de igual magnitud en ambas direcciones [12], para lo cual se cumple que: 


$$
R m=\frac{f_{c u}^{\prime}\left(f^{\prime} t u+a f^{\prime} c p\right)}{U\left(f^{\prime} t u+a f^{\prime} c u\right)}
$$

Donde:

$R_{m}=$ esfuerzo en la falla del murete

$U$ = coeficiente de mayoración, tener en cuenta que la distribución de esfuerzos no es uniforme siempre.

$f^{\prime}{ }^{\prime}=$ resistencia uniaxial a compresión de la unidad

$f^{\prime \prime}=$ resistencia biaxial a tensión de la unidad

$f^{\prime c p}=$ resistencia a la compresión uniaxial del mortero de pega

$\mathrm{a}=\mathrm{j} / 4.1 \mathrm{~h}, \mathrm{j}=$ espesor de la junta, $\mathrm{h}=$ altura del ladrillo

Al hacer algunas simplificaciones como: $\mathrm{j}=10 \mathrm{~mm}, \mathrm{U}=1.5, \mathrm{f}^{\prime}{ }_{\mathrm{tu}}=$ al $10 \% \mathrm{f}^{\prime}{ }_{\mathrm{cu}}$ se obtiene que:

$$
R m=\left(\frac{2 h}{73+3 h}\right) f^{\prime} c u+\left(\frac{49}{73+3 h}\right) f^{\prime} c p
$$

La [4] establece el valor $\mathrm{f}^{\prime}{ }_{\mathrm{m}}$ con base en la calidad de los materiales, cuando no se tienen ensayos de muretes preliminares o históricos, mediante:

$$
\begin{array}{r}
R m=\left(\frac{2 h}{75+3 h}\right) f_{c u}^{\prime}+\left(\frac{50 k_{p}}{75+3 h}\right) f_{c p}^{\prime} \leq 0.8 f^{\prime} c u \\
f_{m}^{\prime}+0.75 R m
\end{array}
$$

Donde:

$\mathrm{f}_{\mathrm{m}}=$ = resistencia nominal a la compresión de la mampostería, MPa

$\mathrm{k}_{\mathrm{p}}=$ factor de corrección por absorción de la unidad, adimensional $\left(k_{\mathrm{p}}=0.8\right.$ para unidades de arcilla cocida)

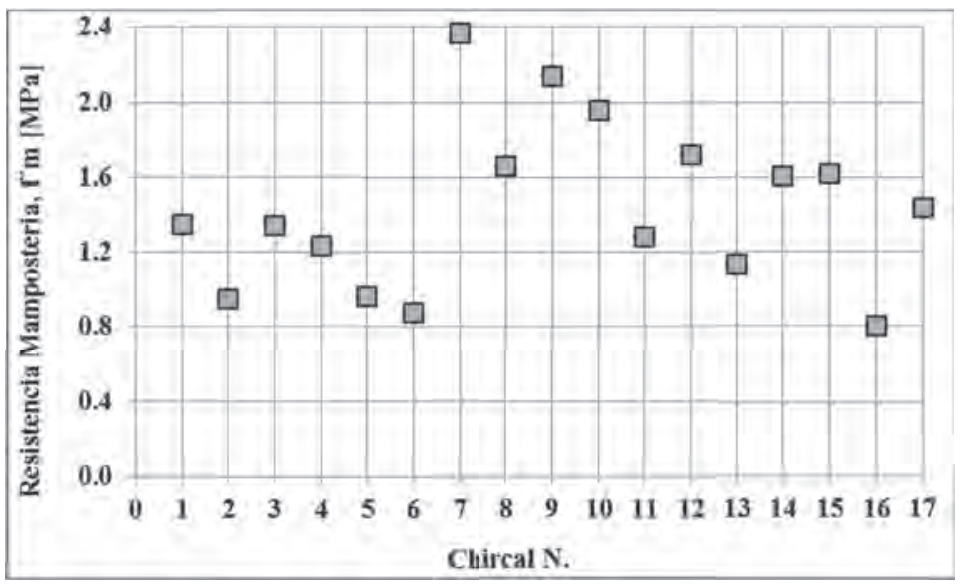

Figura 11. Resistencia nominal a compresión esperada $f^{\prime}{ }_{m^{\prime}}$ Ocaña. [1] 
Es decir, que la resistencia a la compresión de la mampostería f'm, para los ladrillos producidos en esta parte del País, se puede estimar mediante la expresión (7) y (8) y la fluctuación de estos resultados para cada fabricante se observa en la Figura 11, donde la máxima resistencia a la compresión de la mampostería esperada es de 2.37 MPa que corresponde al chircal 7, y la menor resistencia a la compresión de la mampostería esperada es de $0.80 \mathrm{MPa}$ del chircal 16, con un valor promedio para el municipio de Ocaña de 1.44 MPa independiente del tipo de mortero utilizado M o S, pues el aporte del ladrillo a la resistencia a la compresión de la mampostería es baja, debido a la limitante de diseño que sólo permite hasta el 80\% de la resistencia uniaxial a la compresión del ladrillo y es poca la contribución del mortero.

\section{CONCLUSIONES}

De acuerdo con los análisis de granulometría por hidrómetro y análisis granulométrico por tamizado por vía seca realizados al material arcilloso objeto de estudio se puede afirmar que se caracteriza por poseer una alto porcentaje de fracción arenosa y limos equivalente al $81 \%$ y una pobre fracción arcillosa equivalente al 19\% del total de su composición, es decir, son clasificadas como arcillas arenosas inorgánicas con media plasticidad, con índice de plasticidad mayor al 10\%, es decir, son arcillas que permiten el conformado y compactación de las piezas de mampostería.

Además, con el índice de plasticidad por el método de los límites de Atterberg, se estableció que el alto grado de abrasión que ejerce la arena en el material arcilloso, puede ser correlacionado con su baja compactación, teniendo en cuenta que el incremento en la plasticidad de las pastas cerámicas les confiere mayor compactación, resistencia mecánica y los hace más manejables en el proceso de conformado o moldeo de piezas cerámicas.

Los ladrillos producidos en Ocaña, no cumplen las resistencias establecidas para Colombia [15]; la TIA promedio es de $0.387 \mathrm{~g} / \mathrm{cm}^{2} / \mathrm{mín}$, indica que las piezas de ladrillos deberán tener un prehumedecimiento mínimo de 24 horas y la AF promedio es de $17.41 \%$, apropiado para muros interiores, pero excede en $4.41 \%$ el valor máximo para su utilización en muros exteriores porque su absorción máxima permitida es de 13\% [15].

No se encontró una relación que permita estimar una expresión entre la TIA y la resistencia a la compresión del ladrillo macizo de arcilla cocida, como también entre la AF y la resistencia a la compresión del ladrillo, pues sus coeficientes de correlación (R) fueron de 0.032 y 0.037 respectivamente, resultados diferentes a los obtenidos en otras investigaciones [1 1] donde para $f^{\prime}{ }_{c u}$ Vs. TIA el $R=-0.77$ y para $f^{\prime}{ }_{c u}$ Vs. AF es $R=-0.24$. Estos resultados pueden ser producto de la variabilidad del proceso de cocción, puesto que no se puede definir con claridad los ciclos de cocción empleados por los fabricantes en la producción de ladrillos tolete macizos, tomando como referencia que la temperatura promedio no alcanza los $850^{\circ} \mathrm{C}$ en la mejor posición dentro del horno, pues hay zonas donde la temperatura escasamente alcanza los $250^{\circ} \mathrm{C}$ (tomada con termocuplas tipo K EXTECH) [10]. 
La resistencia nominal a la compresión de muros en ladrillo macizos de arcilla cocida tiene valores que oscilan entre 0.8 hasta 2.4 MPa y un valor promedio para el municipio de Ocaña de 1.44 MPa lo cual es bajo, si lo comparamos con la especificación de resistencia de 14 MPa para ladrillos macizos [15] y su restricción de usar sólo el 80\% de su resistencia [4], es decir, una resistencia a la compresión de la mampostería de $8.4 \mathrm{MPa}$, lo cual indica que en Ocaña, la resistencia de la mampostería a la compresión está entre el 9.5 al 28.6\% del mínimo esperado, representando al menos, un alto grado de vulnerabilidad a la compresión en muros de ladrillo tolete macizo, usados principalmente en viviendas construidas con el sistema de resistencia sísmica de mampostería confinada. Esto indica la necesidad de implementar hornos comunitarios que garanticen la temperatura de sintetización del material, un mejoramiento en el proceso de producción de las piezas de mampostería ya sea mediante extrucción u otro medio, y el diseño de mezclas de arcilla que permitan un mejoramiento de las propiedades mecánicas.

Se ha observado en los alrededores de la Ciudad, muros con fisuras y grietas causadas por solicitaciones de resistencias a la compresión y corte que indican una falla local que disminuye la capacidad global del sistema de resistencia sísmica y hace necesario que las entidades gubernamentales tengan en cuenta estas viviendas, para delimitar zonas potenciales de riesgo y posibles reforzamientos, mediante mampostería reforzada exterior sugerida en la Norma Sismo Resistente Colombiana (sección D.12) [4].

\section{REFERENCIAS BIBLIOGRÁFICAS}

[1] Afanador G.N., (2011). Proyecto de investigación “Propiedades mecánicas y físicas de los ladrillos producidos en el municipio de Ocaña". Universidad Francisco de Paula Santander, Ocaña.

[2] Alcaldía municipal de Ocaña, (2011). Plan de Ordenamiento Territorial (POT). Ocaña, Norte de Santander.

[3] Asociación de Ingeniería Sísmica (AIS)., (2004). Guía de Patologías constructivas, estructurales y no estructurales. Bogotá.

[4] Asociación Colombiana de Ingeniería sísmica (AIS)., (2010). Reglamento colombiano de construcción sismo resistente. NSR-10. Bogotá.

[5] Birnbaum Z.W., and Saunders S.C., (1969). A new family of life distributions. In: Journal of applied probability. Vol.6, pp. 319-327.

[6] Cuéllar E., Portillo J., Renderos M., y Vides F., (2006). Evaluación de la resistencia a la fractura de los ladrillos de barro fabricados por compresión. Trabajo de grado no publicada, Universidad Centroamericana José Simeón Cañas. San Salvador. 
[7] Espinosa E., y Cantú M., (2010). Caracterización y aplicación de la distribución Birnbaum Saunders como modelo de tiempo de vida. En: Revista Agraria -Nueva Época- Año XVII. Vol. 52 (1), pp. 19-27.

[8] Gallegos H., y Casabonne C., (2005). Albañilería estructural. Pontificia Universidad Católica del Perú, Lima.

[9] Gere M.G. and Goodno B.J., (2011). Mechanics of materials. Cengage learning. United States.

[10] Guerrero G., (2011). Proyecto de investigación: Eficiencia energética y uso racional de la energía en la producción de material cerámico en la región. Universidad Francisco de Paula Santander, Ocaña.

[11] Kaushik Hemant B., Durgesh C. Rai and Sudhir K. Jain., (2007). Stress-strain characteristics of clay brick masonry under uniaxial compression. In: Journal of materials in civil engineering ASCE. pp. 728-739.

[12] Hilsdorf H.K., (1969). An investigation into the failure mechanism of brick masonry under axial compression in designing. In: Engineering and constructing with masonry products. Houston, pp. 34-41.

[13] Instituto Colombiano de Normas Técnicas (NTC)., (1999). Suelos. Ensayos para determinar la granulometría por tamizado (NTC 1522). 1ra ratificación. Bogotá.

[14] Instituto Colombiano de Normas Técnicas (NTC)., (1999). Método de ensayo para la determinación del límite líquido, del límite plástico y del índice de plasticidad de los suelos cohesivos (NTC 4603). 1 ra actualización. Bogotá.

[15] Instituto Colombiano de Normas Técnicas (NTC)., (2000). Unidades de mampostería de arcilla cocida. Ladrillos y bloques cerámicos (NTC 4205). 1 ra actualización. Bogotá.

[16] Instituto Colombiano de Normas Técnicas (NTC)., (2005). Métodos para muestreo y ensayos de unidades de mampostería y otros productos de arcilla (NTC 4017). 1ra actualización. Bogotá.

[17] Instituto Nacional de Vías., (2007). Procedimiento para la preparación de muestras de suelos por cuarteo, (INV E-014-07). Bogotá, 6 p.

[18] Instituto Nacional de Vías., (2007). Análisis granulométrico por medio del Hidrómetro (INV E-124-07). Bogotá, 20 p. 
[19] Palacios L. H., (2010). Calculo de los parámetros de la distribución de Weibull, En: http:// confiabilidad.net/articulos/calculo-de-los-parametros-de-la-distribucion-de-weibull/ (agosto de 20111 .

[20] Takeuchi C., (2007). Comportamiento en la mampostería estructural. Universidad Nacional de Colombia, Bogotá. 